\title{
Implicit effects of emotional contexts: An ERP study
}

\author{
Antonio Jaeger • Michael D. Rugg
}

Published online: 15 July 2012

(C) Psychonomic Society, Inc. 2012

\begin{abstract}
Previous recognition memory experiments have demonstrated that the ERPs elicited by correctly recognized test items differ according to whether the items were encoded in an emotionally arousing or an emotionally neutral context. It is not clear, however, whether these ERP differences depend on the explicit recognition of the items. We addressed this question in the present study by contrasting the ERPs elicited by test items encoded in emotionally negative or emotionally neutral study contexts, according to whether the items were correctly recognized or misclassified as new. Recognized items associated with emotional rather than neutral contexts elicited an early positive-going and a later negative-going effect that resembled the effects reported in prior studies. Relative to unrecognized items encoded in neutral contexts, unrecognized items encoded in emotional contexts elicited a sustained, frontal-maximum, positive-going effect that onset at about $200 \mathrm{~ms}$ poststimulus. This effect may reflect an influence of emotional arousal on the neural correlates of implicit memory.
\end{abstract}

Keywords Episodic memory $\cdot$ Implicit memory $\cdot$ Emotion · ERP · Priming

A. Jaeger

Department of Psychology, Washington University,

St Louis, MO, USA

\section{A. Jaeger $(\bowtie)$}

Universidade Federal do Rio Grande do Sul, Departamento de Psicologia do Desenvolvimento e da Personalidade, Instituto de Psicologia, Rua Ramiro Barcellos 2600, 90035-003 Porto Alegre-RS, Brazil

e-mail: antonio.jaeger@gmail.com

\section{D. Rugg}

Center for Vital Longevity and School of Behavioral and Brain Sciences, University of Texas at Dallas,

Dallas, TX, USA
Considerable evidence has indicated that memory is enhanced for emotionally arousing relative to unarousing events (Christianson, 1992; Kensinger, 2007). Experiments investigating the neural mechanisms underlying this phenomenon have focused primarily on consolidation processes (Hamann, 2001), but recent neuroimaging and event-related potential (ERP) studies have investigated the effects of emotional arousal at the time of retrieval (Buchanan, 2007). Several of these studies have focused on information encoded in association with emotional contexts (Jaeger, Johnson, Corona, \& Rugg, 2009; Maratos, Dolan, Morris, Henson, \& Rugg, 2001; Maratos \& Rugg, 2001; Smith, Dolan, \& Rugg, 2004; Smith, Henson, Rugg, \& Dolan, 2005; Smith, Stephan, Rugg, \& Dolan, 2006).

One such study (Smith et al., 2004; see also Maratos \& Rugg, 2001) demonstrated that ERPs elicited during recognition of objects that had been studied in association with emotional versus nonemotional pictorial contexts differed in two distinct ways. The first effect comprised a relatively earlyonsetting (circa 300-500 ms) temporal-maximum positivity for objects encoded in emotional versus neutral contexts. The second effect, which also took the form of a positive-going shift in the ERPs for emotionally encoded objects, had a relatively late onset (circa $700 \mathrm{~ms}$ ), was frontally distributed, and persisted for several hundred milliseconds.

The results reported by Smith et al. (2004) were extended by Jaeger et al. (2009), who contrasted ERPs elicited by recognized items that had been studied in association with emotionally negative versus emotionally neutral contexts either $10 \mathrm{~min}$ or $24 \mathrm{~h}$ earlier. In line with the findings of Smith et al. (2004), Jaeger et al. reported that after the shorter studytest delay, recognized items from emotional contexts elicited an early (circa $200 \mathrm{~ms}$ poststimulus) positivity, relative to items from neutral contexts. However, unlike the results reported by Smith et al. (2004), this positivity was sustained until about 1,100 ms post-stimulus-onset, rather than 
demonstrating distinct early and late phases. After the 24h study-test delay, the effects of emotional context were reversed in polarity (i.e., items from emotional contexts elicited a more negative waveform than did items from neutral contexts) and were maximal over the posterior scalp. The polarity differences between short and long study-test delays were interpreted as evidence that emotion-specific consolidation mechanisms modulate the influence of emotion on retrieval-related neural activity.

Importantly, the onset latencies of the emotional context effects reported by Smith et al. (2004) and Jaeger et al. (2009) were similar to the onsets of ERP differences found for directly presented emotional and nonemotional items (Cuthbert, Schupp, Bradley, Birbaumer, \& Lang, 2000; Keil, Bradley, Hauk, Rockstroh, Elbert, Lang, 2002; Schupp, Cuthbert, Bradley, Cacioppo, Ito, Lang, 2000). The similarity of these onset latencies was interpreted as evidence that the objects associated with emotional contexts acquired the arousing properties of the contexts in which they had been studied, such that the items became (at least temporarily) emotionally arousing (Jaeger et al., 2009; Smith et al., 2004). If this is the case, one might expect the early-onsetting differences between test items studied in emotionally arousing versus nonarousing contexts to be independent of whether the items were explicitly recognized or misjudged as new. That is, the effects should not depend on whether information about the study context was successfully retrieved.

Alternatively, it may be that the above-mentioned early ERP effects reflect the influence of emotion on retrieval. If so, different patterns of effects might be expected, depending on whether or not retrieval was successful. By one scenario, the effects might be present only when recognition is successful, linking the effects to explicit retrieval of information from the study episode. By another scenario, dissociable effects might be evident for recognized and unrecognized test items, suggesting that the effects of emotional study contexts separately modulate explicit and implicit retrieval mechanisms.

Although prior studies have investigated implicit emotional memory (Christianson, 1992; Eder, Leuthold, Rothermund, \& Schweinberger, 2012; Hinojosa, Carretié, Méndez-Bértolo, Míguez, \& Pozo, 2009; Li, Zinbarg, Boehm, \& Paller, 2008; Tapia, Carretié, Sierra, \& Mercado, 2008), these studies employed emotionally arousing stimuli and short study-test intervals $(<1 \mathrm{~h})$. In the present study, we investigated the neural correlates of explicit and implicit retrieval over a markedly longer study-test interval by contrasting the ERPs elicited by recognized and unrecognized (missed) test items that had been studied $24 \mathrm{~h}$ earlier in either emotionally arousing or neutral contexts.

We employed an experimental procedure initially introduced by Maratos and Rugg (2001) for the study of the incidental retrieval of emotionally arousing verbal information, and which was later modified by Smith et al. (2004) for use with pictorial materials. The 24 -h study-test interval employed here was useful to ensure that a sufficient proportion of the studied items would be forgotten at test, permitting the key experimental contrasts to be undertaken (see also Jaeger et al., 2009). We employed emotionally negative and neutral study contexts, but did not include emotionally positive backgrounds. The rationale for excluding the latter contexts was that negatively valenced materials are, in general, more arousing than positively valenced materials, and hence provide a more powerful contrast with neutral information (see Lang, Bradley \& Cuthbert, 2008). Additionally, prior findings have suggested that the effects of emotional context on the ERP correlates of retrieval success do not differ as a function of valence (Smith et al., 2004).

If the interpretation of the early-onsetting effects of emotional context at retrieval given by Jaeger et al. (2009) and Smith et al. (2004) is correct-that is, if the effects reflect the emotionally arousing properties of the items themselves - then the timings and scalp distributions of these effects should be equivalent for recognized and unrecognized items. If the effects depend on the explicit retrieval of study information, they should be confined to correctly recognized items. Finally, if emotional context can modulate implicit as well as explicit retrieval processes, qualitatively different effects should be expected, according to whether the test item is recognized or unrecognized.

\section{Method}

\section{Participants}

A group of 21 individuals (11 female, 10 male) between 18 and 29 years old (mean age $=19.9$ years) participated in return for payment of $\$ 15 / \mathrm{h}$. The participants were recruited from the University of California, Irvine (UCI), community, were right-handed, and were native English speakers with normal or corrected-to-normal vision. Informed consent was obtained in accordance with UCI Institutional Review Board guidelines. Of these 21 participants, one was rejected from the analysis due to excessive eye movement artifacts. The sample over whom data were analyzed comprised 11 female and 9 male participants.

\section{Stimulus materials}

The stimuli were similar to those employed by Smith et al. (2004). They consisted of a pool of 300 pictures of emotionally neutral objects (critical items) and a pool of 200 background contexts that varied in their emotional arousal and valence. The contexts subtended maximum vertical and 
horizontal angles of $12.2^{\circ}$ and $19.2^{\circ}$, respectively, at the $1-\mathrm{m}$ viewing distance. The critical items (maximum visual angle $5.7^{\circ} \times 6.8^{\circ}$ ) were presented within a white box (subtending $7.5^{\circ} \times 7.5^{\circ}$ ) that demarcated their separation from the contexts during study and from the monitor background at test (see Fig. 1). Approximately $50 \%$ of the contexts were taken from the International Affective Picture System (IAPS; Lang et al., 2008), which consists of a series of pictures standardized for arousal and emotional valence. The remaining contexts were obtained from a variety of Internet sources. All of the contexts were rated for arousal and valence by 10 North American participants ( 5 female, 5 male; mean age 22 years) who did not participate in the ERP experiment. The ratings were acquired with two 5-point Likert scales that assessed arousal $(1=$ very calming, $5=$ very arousing $)$ and valence $(1=$ very negative, $5=$ very positive $)$. The contexts selected as experimental materials consisted of 100 highly arousing emotionally negative pictures (for arousal, $M=4.46, S D=0.66$; for valence, $M=1.44, S D=$
0.62 ) and 100 moderately arousing neutral pictures (arousal, $M=2.98, S D=0.79$; valence, $M=3.36, S D=0.73$ ). No positively valenced pictures were included because such pictures are in general less emotionally arousing than negatively valenced pictures (see Lang et al., 2008), and prior research had shown that ERP effects of emotion do not differ as a function of valence at retrieval (see the introduction). Most of the selected contexts were scenes that contained people ( $82 \%$ of the emotionally negative and $74 \%$ of the emotionally neutral contexts). The remaining contexts depicted a mixture of animals, landscapes, and objects. None of the contexts depicted sexual material. The set of critical items (e.g., ball, chair, and guitar) belonged to a variety of different semantic categories and had been confirmed as neutrally valenced in a pilot study conducted by Smith et al. (2004). The stimuli were randomly allocated to experimental conditions on a participant-specific basis. During the encoding phase, the 200 contexts were presented paired with 200 critical items. The remaining 100 items
Fig. 1 Schematic view of the study and test phase stimulus presentations

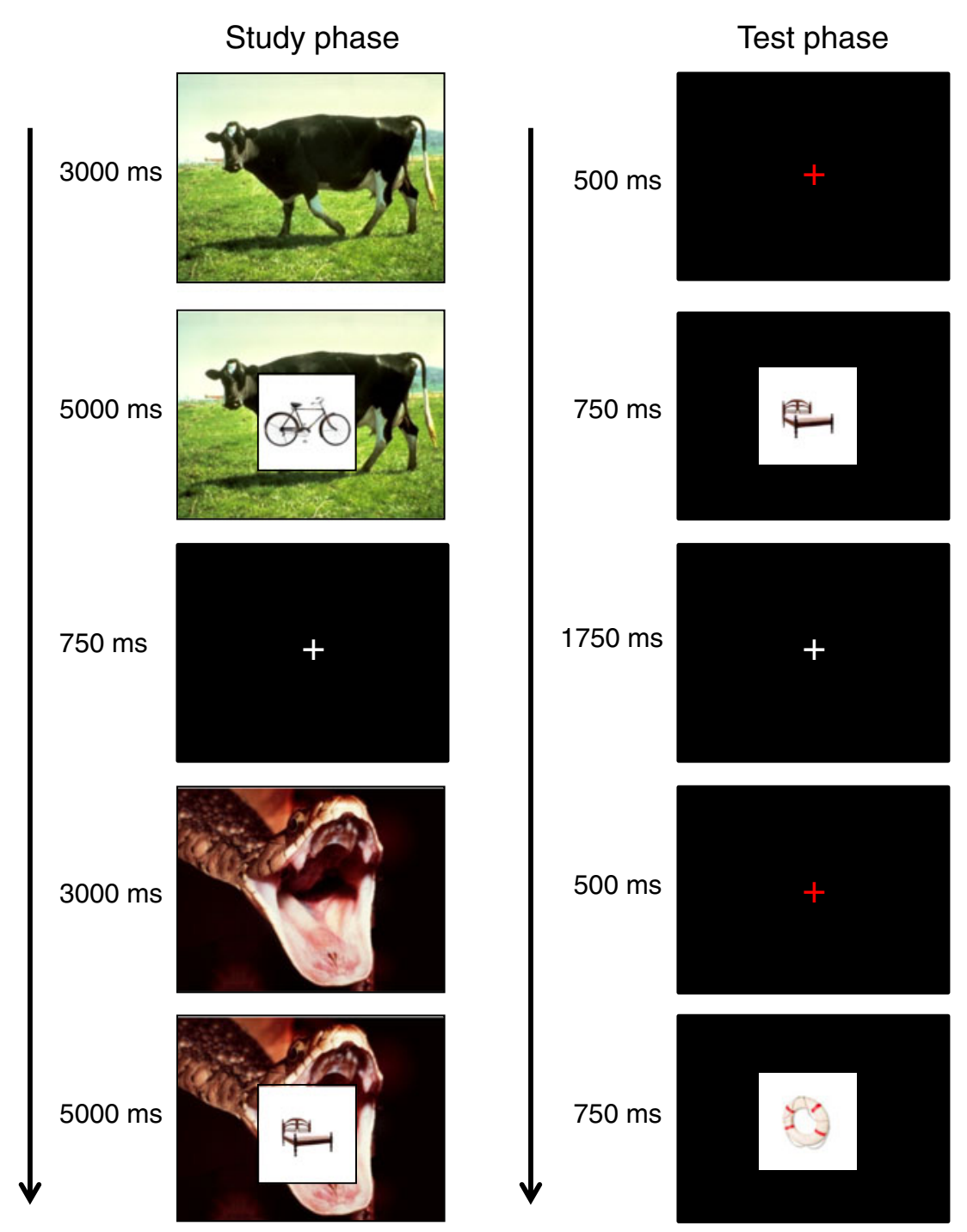


provided the "new" items at test. The presentation order of each class of context at the study phase was pseudorandomized, with no more than three contexts from the same category presented consecutively.

\section{Study procedure}

As is shown in Fig. 1, on each study trial a white fixation cross was initially presented against a black background for $750 \mathrm{~ms}$, serving as a warning for the upcoming presentation of a context. Each context was presented for $3 \mathrm{~s}$, during which the participants verbally rated its emotional valence [from -2 (very negative) to +2 (very positive)]. The study item was then superimposed across the center of the context, after which the stimulus pairing was presented for $5 \mathrm{~s}$. During this period, participants were required to "visualize a connection" between the two figures. The white fixation cross was then re-presented, signaling the onset of a new trial. The stimulus onset asynchrony (SOA) was thus $8,750 \mathrm{~ms}$. A practice study session, administered prior to the study session proper, consisted of six trials wherein the participants were required to verbally describe the connection that they had been made between the items and the contexts. Rest breaks were given after each 52 trials in the study session proper.

\section{Test procedure}

The test phase followed the encoding phase $24 \mathrm{~h}$ later. On each trial, a red fixation cross was presented against a black background for $500 \mathrm{~ms}$, signaling that the critical item was about to be shown (see Fig. 1). Each item was presented for $750 \mathrm{~ms}$, immediately followed by a white fixation cross against a black background that was presented for $1,750 \mathrm{~ms}$. The subsequent presentation of a red fixation cross signaled the onset of a new trial, creating an SOA of $3 \mathrm{~s}$. The participants kept their left and right forefingers resting on a response pad. They were instructed to respond by pressing one button when they judged that a test item had been presented in the encoding phase and another button when the item was judged to be new. The assignments of the left and right response buttons to the two types of judgments were counterbalanced across participants. Rest breaks were provided after every 102 trials, with the first two trials of each block being fillers items. During the practice phase, six items from the practice of the study phase and four new items were presented. The test phase proper lasted about $30 \mathrm{~min}$.

\section{ERP recording and analysis}

Electroencephalographic activity (EEG) was recorded continuously from 31 silver/silver-chloride electrodes. Twenty-nine of these electrodes were embedded in an elastic cap with additional electrodes affixed to each mastoid process. The locations of the cap electrodes were based on the International 10-20 system (American Electroencephalographic Society, 1994) and corresponded to midline sites (Fz, Cz, and $\mathrm{Pz}$ ) as well as homotopic (left/right) pairs of sites (Fp1/Fp2, AF7/AF8, F3/F4, F5/F6, F7/F8, C3/ C4, C5/C6, T7/T8, P3/P4, P5/P6, P7/P8, PO7/PO8, and O1/ $\mathrm{O} 2)$. Vertical and horizontal electro-oculograms (EOG) were recorded from bipolar electrode pairs situated above and below the left eye and on each outer canthus. Data were acquired with Contact Precision amplifiers (London, U.K.) at a 256-Hz sampling rate and an amplifier bandwidth of $0.01-40 \mathrm{~Hz}$ $(-3 \mathrm{~dB})$. Interelectrode impedances were adjusted to below 5 $\mathrm{k} \Omega$. Offline, the continuous EEG data were epoched to a 2,048-ms duration with a $102-\mathrm{ms}$ prestimulus baseline. The resulting epochs were down-sampled to a $125-\mathrm{Hz}$ sampling rate and algebraically re-referenced to linked mastoids. Trials containing movement artifact, EOG artifacts other than blinks, or excessive baseline drift were rejected. The averaged ERPs were smoothed with a five-point moving-window filter at a cutoff of $19.4 \mathrm{~Hz}(-3 \mathrm{~dB})$. A previously described linear regression method (Henson, Rylands, Ross, Vuilleumier, \& Rugg, 2004) was used to correct blink artifacts for all participants.

\section{Results}

\section{Behavioral data}

The mean accuracy and reaction times (RTs) for studied items paired with emotionally negative contexts (emotional hits and misses) and emotionally neutral contexts (neutral hits and misses), as well as those data for correctly rejected new items, are shown in Table 1. An analysis of variance (ANOVA) of the accuracy data (with Item Type as a factor) gave rise to a significant item type effect, $F(1.1,21.4)=$ 18.17, $p<.001$ (here and in all subsequent ANOVAs, the degrees of freedom associated with effects involving factors with more than two levels were corrected for nonsphericity by the Greenhouse-Geisser procedure). As was revealed by follow-up $t$ tests, participants were more accurate in correctly rejecting new items than in correctly endorsing old items studied in either emotionally negative or neutral contexts $[t \mathrm{~s}$

Table 1 Means (and $S D$ s) for the accuracies and reaction times (RTs) of responses to remembered and missed emotional, neutral, and new items

\begin{tabular}{llllll}
\hline & $\begin{array}{l}\text { Emotional } \\
\text { Hit }\end{array}$ & $\begin{array}{l}\text { Neutral } \\
\text { Hit }\end{array}$ & $\begin{array}{l}\text { Emotional } \\
\text { Miss }\end{array}$ & $\begin{array}{l}\text { Neutral } \\
\text { Miss }\end{array}$ & New \\
\hline $\begin{array}{l}\text { Accuracy } \\
\text { RT }\end{array}$ & $0.50(0.19)$ & $0.51(0.18)$ & $0.50(0.18)$ & $0.49(0.18)$ & $0.91(0.07)$ \\
& $1,086(226)$ & $1,062(226)$ & $1,071(243)$ & $1,093(245)$ & $1,033(221)$ \\
\hline
\end{tabular}


$(19)=7.70$ and 7.89, respectively, $p \mathrm{~s}<.001]$. A further $t$ test revealed no effect of study context $[t(19)=0.71, p=.48]$. A one-way ANOVA of the RTs associated with the five response categories revealed no significant effect $[F(1.8,34.2)$ $=2.79, p>.08]$. Analysis of the response bias index $\mathrm{Br}$ revealed that there was no difference in criteria according to whether the items had been paired with emotional or neutral contexts $(\mathrm{Br}=0.15$ for both conditions). $\mathrm{Br}$ values below 0.5 are indicative of a conservative response bias (Snodgrass \& Corwin, 1988); hence, participants adopted an equally conservative criterion, regardless of encoding context.

\section{ERP data}

ERPs were quantified by measuring the mean amplitudes (relative to the mean of the 102-ms prestimulus baseline) of four consecutive latency intervals $(200-300,300-500,500-800$, and $800-1,100 \mathrm{~ms})$. We selected the latency regions in which Jaeger et al. (2009) showed effects of emotion (200-300, 300500 , and 500-1,100 ms post-stimulus-onset) and subdivided those so as to isolate the regions in which "midfrontal" and "parietal" retrieval old-new effects are typically reported (i.e., 300-500 and 500-800 ms, respectively; Rugg \& Curran, 2007). From the findings reported by Jaeger et al., we expected that the effects of emotion elicited by recognized items would be evident in the earliest latency region (i.e., 200-300 ms) and would continue through subsequent latency regions. Given the early onset of the implicit-memory effects on ERPs reported in prior studies (e.g., Voss, Schendan, \& Paller, 2010), we anticipated that any ERP effects differentiating recognized and unrecognized test items would be evident from around $200 \mathrm{~ms}$ post-stimulus-onset. Statistical analyses were conducted on the data derived from the electrode sites depicted in Fig. 2. For each item type, the mean numbers (and ranges) of trials that contributed to the waveforms were 40 (17-71) and 41 (20-69) for emotionally negative and neutral hits, respectively; 40 (18-70) and 38 (16-66) for emotionally negative and neutral misses, respectively; and 70 (45-88) for correct rejections.

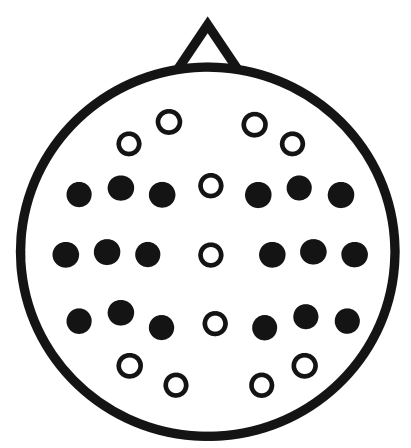

Fig. 2 Electrode arrangement and sites employed for analyses of the mean amplitudes (black in-fills)
The analysis of ERPs was conducted in three stages. First, the differences in amplitude of the ERPs elicited by correctly classified old items and by correctly rejected new items were contrasted in order to investigate old-new effects as a function of the emotional status of the old item (emotional vs. neutral study context). Second, the ERPs elicited by studied items misclassified as new (misses) and by correctly rejected items were contrasted in order to explore emotional memory effects in the absence of explicit recognition. Third, differences between emotional versus neutral hits and emotional versus neutral misses were contrasted in order to identify potential dissimilarities in the emotional effects for explicitly and implicitly retrieved items. In each case, analyses of the scalp topographies of the relevant contrasts were also undertaken. The grand average ERPs elicited by hits and misses - respectively - contrasted with correct rejections are shown in Figs. 3 and 4.

Analysis of the ERPs elicited by hits and correct rejections

As can be seen in Fig. 3, the ERPs elicited by hits and correct rejections begin to differ from approximately $200 \mathrm{~ms}$ poststimulus. Hits for both emotional item types appear to elicit a more positive-going waveform than do correct rejections. These differences are frontally distributed and left-lateralized over posterior electrode sites. Divergences between the two classes of hits are apparent from around $800 \mathrm{~ms}$ poststimulus, taking the form of a positive-going shift in the ERPs elicited by neutral relative to emotional items, particularly over the left hemisphere.

The data analysis involved two stages. In the first stage, an overall ANOVA was performed, factored according to Item Type (emotional hits, neutral hits, or correct rejection), Hemisphere, Chain (frontal, central/temporal, or parietal), and Lateral Site (inferior, middle, or superior). For each latency region in which the overall ANOVA revealed reliable main effects of item type or interactions between item type and another factor or factors, subsidiary ANOVAs were conducted that separately contrasted each class of hit (negative and neutral) to the correct-rejection waveforms. Further subsidiary ANOVAs were performed as necessary to identify electrode clusters where the emotion and oldnew effects were most in evidence. The outcomes of the initial ANOVAs are shown in Table 2. As can be seen in the table, there were interactions between the factor Item Type and various topographic factors in the 200- to 300-, 300- to 500-, 500- to 800-, and 800- to 1,100 -ms latency windows.

\section{Neutral hit versus correct rejection}

Table 2 shows that a main effect of item type was evident for the last three latency regions. As is demonstrated by the interactions involving anterior-posterior location and 
Fig. 3 Grand average waveforms elicited by emotional hits, neutral hits, and correct rejections. The locations of the depicted sites are indicated in the inset
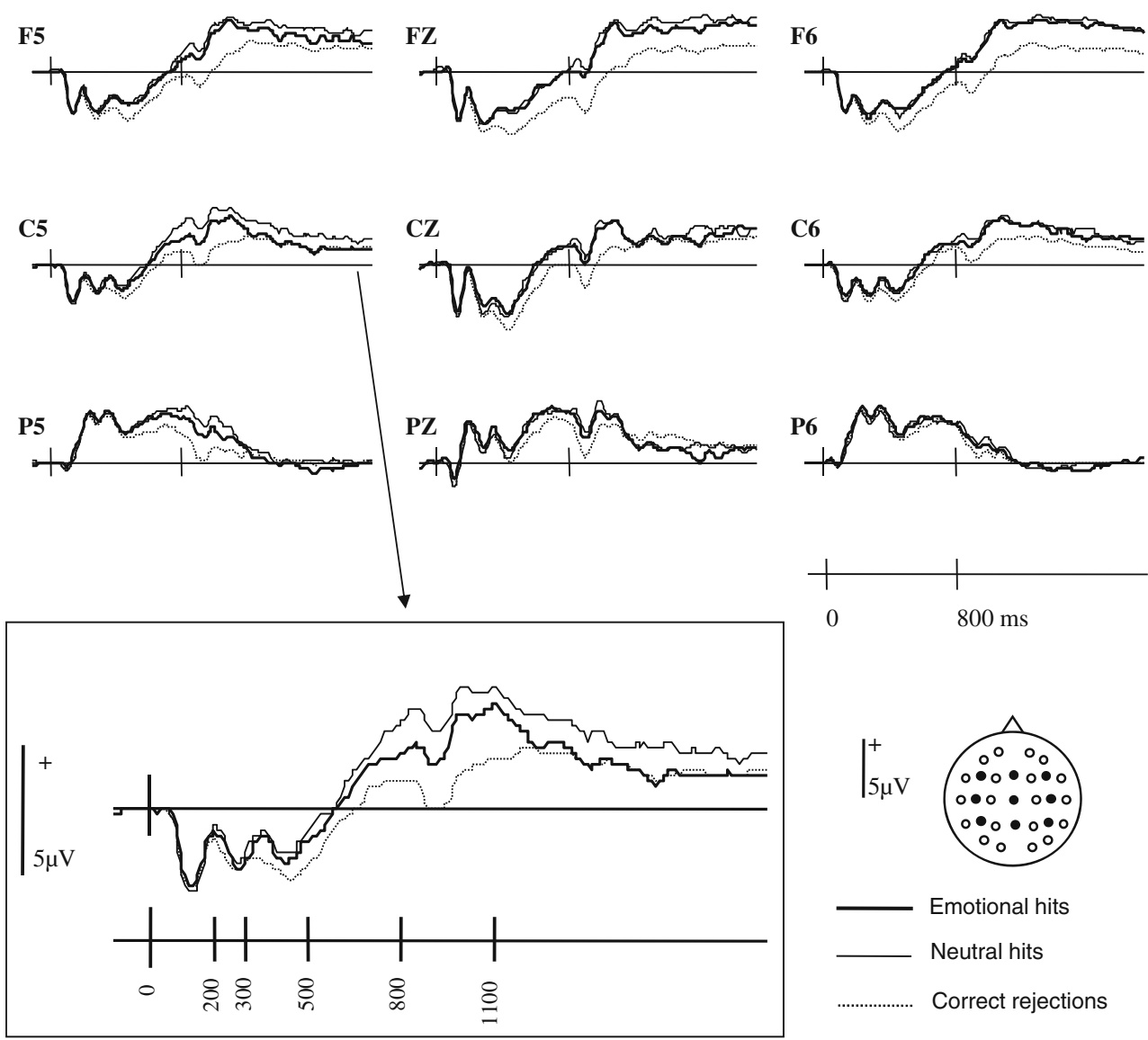

Emotional hits

Neutral hits

Correct rejections hemisphere, for all latency regions, neutral hits elicited a more positive waveform over the frontal scalp than did correct rejections. This positivity was also found over the left posterior electrodes for the last two latency regions (see Fig. 3). Follow-up ANOVAs restricted to the electrodes typically associated with the explicit retrieval processes of familiarity and recollection (frontal chain and left parietal chain for the 300 - to $500-\mathrm{ms}$ and 500 - to $800-\mathrm{ms}$ ranges, respectively) revealed reliable main effects of item type in which neutral hits elicited more positive waveforms than did correct rejections [frontal, $F(1,19)=14.42, p<.001$; parietal, $F(1,19)=13.32, p<.002]$.

\section{Emotional hit versus correct rejection}

A main effect of item type was found for the later three epochs. Interactions involving hemisphere, anterior-posterior location, and site indicated that emotional hits elicited more positive-going effects than did correct rejections. These effects were elicited in all latency ranges and were most prominent in frontal electrodes. This positive shift was also evident over the posterior sites from $\sim 500 \mathrm{~ms}$ onward. Follow-up ANOVAs restricted to frontal electrodes for the 300- to 500-ms epoch and left parietal electrodes for the 500- to 800-ms epoch yielded main effects of item type [frontal, $F(1,19)=11.17, p<.003$; parietal, $F(1,19)=$
$5.66, p<.03]$. In both regions, emotional hits elicited a more positive waveform than did correct rejections.

\section{Emotional versus neutral hits}

Reliable interactions involving item type and site, hemisphere, and anterior-posterior location were found for the 200- to 300-ms latency region, although no main effects were found when electrodes from the frontal, central, and parietal chains were separately submitted to follow-up ANOVAs. For the 800- to 1,100-ms epoch, an interaction between item type and hemisphere indicated that emotional items elicited a negative shift relative to neutral items. The latter effect was significant over left central $[F(1,19)=5.91, p<.02]$ and left parietal $[F(1,19)=4.95, p<.04]$ sites (see Fig. 3).

Analysis of ERPs elicited by misses and correct rejections

As can be seen in Fig. 4, the ERPs elicited by misses and correct rejections begin to differ from approximately $200 \mathrm{~ms}$ poststimulus. This divergence seems to be driven primarily by emotional misses, which appear to elicit a more positivegoing waveform over frontal sites relative to both neutral misses and correct rejections.

Quantification of these data was accomplished by measuring the mean amplitudes of the 200- to 300-ms, 300- to 500- 
Fig. 4 Grand average waveforms elicited by emotional misses, neutral misses, and correct rejections. The depicted site locations are indicated in the inset

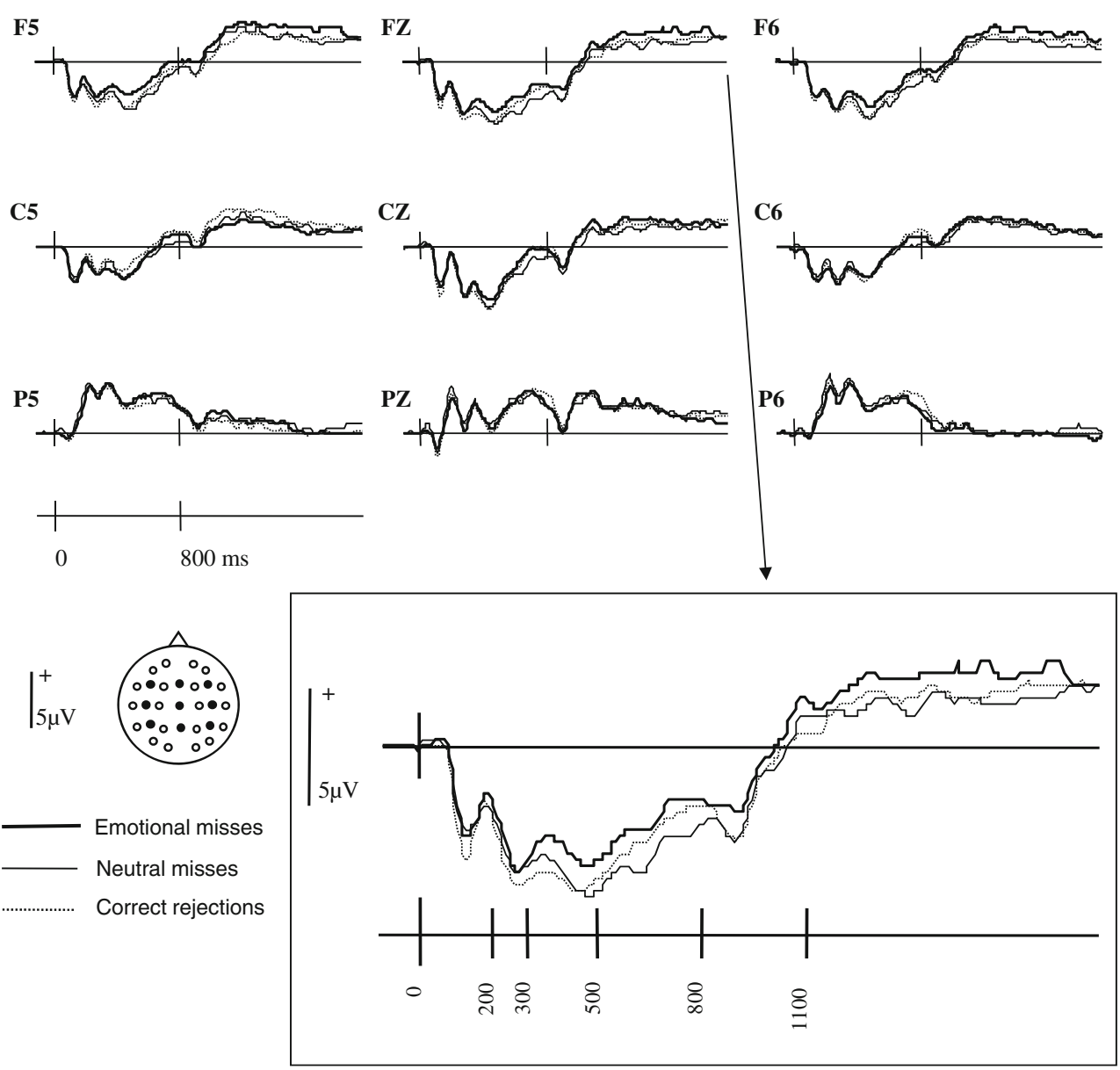

ms, 500- to 800-ms, and 800- to 1,100-ms latency windows. The selection of these latencies was again based on inspection of the waveforms and on the time courses of effects obtained in previous studies (Jaeger et al., 2009; Maratos \& Rugg, 2001; Rugg, Mark, Walla, Schloerscheidt, Birch, Allan, 1998; Smith et al., 2004). Analyses of these data followed the same strategy that was employed above for emotional and neutral hits, and the outcomes of the ANOVAs are shown in Table 3.

As is evident from the table, we found interactions between the factor Item Type and various topographic factors in the 200- to 300-, 300- to 500-, 500- to 800-, and 800- to 1,100-ms latency regions.

\section{Neutral miss versus correct rejection}

As can be seen in Table 3, an interaction involving item type and hemisphere in the 800 - to 1,100 -ms epoch indicated that while neutral misses elicited a waveform slightly more positive than that of correct rejections over the left hemisphere, the opposite pattern was elicited in the right hemisphere, where correct rejections were more positive. Subsidiary ANOVAs conducted separately on the electrodes of each hemisphere, however, failed to show any significant effect $(F<1)$. There were no further interactions involving these two factors.

\section{Emotional miss versus correct rejection}

A reliable item type main effect was evident for the 300- to 500-ms latency region, and interactions involving item type, hemisphere, and anterior-posterior location were revealed in all latency regions (see Table 3). These effects indicate that emotional misses elicited more positive waveforms than did correct rejections over frontal electrodes. Follow-up ANOVAs conducted separately on the data from frontal, central, and parietal chains revealed main effects over the frontal chain for three latency ranges [200-300 ms, $F(1,19)$ $=5.81, p<.02 ; 300-500 \mathrm{~ms}, F(1,19)=22.83, p<.0001$; and $800-1,100 \mathrm{~ms}, F(1,19)=10.41, p<.004]$ and over the central chain for the 300 - to $500-\mathrm{ms}$ latency range $[F(1,19)$ $=4.89, p<.05]$, indicating that emotional misses elicited a significantly more positive waveform at all of these sites relative to correct rejections.

\section{Emotional versus neutral misses}

As can be seen in Table 3, reliable interactions involving item type and anterior-posterior location were found for the three earliest latency regions. The interactions indicated that emotional misses elicited a more positive waveform than did 
Table 2 Contrast of event-related potentials (ERPs) between hits and correct rejections (CRs) at different topographic sites and for different latency regions

\begin{tabular}{|c|c|c|c|c|}
\hline \multirow[t]{2}{*}{ Effect } & \multicolumn{4}{|l|}{ Latency Regions (ms) } \\
\hline & $200-300$ & $300-500$ & $500-800$ & $800-1,100$ \\
\hline \multicolumn{5}{|c|}{ All Hit Versus CR } \\
\hline IT & & $F(1.7,32.1)=3.63, p<.05$ & $F(2,37.8)=5.52, p<.009$ & $F(2,38)=22.71, p<.001$ \\
\hline $\mathrm{IT} \times \mathrm{HM}$ & & & & $F(1.5,28.6)=5.74, p<.02$ \\
\hline $\mathrm{IT} \times \mathrm{AP}$ & & $F(2.2,41)=5.67, p<.007$ & & $F(2.4,44.7)=11.04, p<.001$ \\
\hline $\mathrm{IT} \times \mathrm{ST}$ & $F(2.1,39.6)=3.32, p<.05$ & & $F(2,37.2)=8.24, p<.002$ & $F(1.9,35.6)=11.91, p<.001$ \\
\hline $\mathrm{IT} \times \mathrm{HM} \times \mathrm{AP}$ & $F(2.7,52.1)=4.95, p<.006$ & & $F(2.3,43.6)=3.65, p<.03$ & $F(2.1,40)=3.64, p<.04$ \\
\hline $\mathrm{IT} \times \mathrm{HM} \times \mathrm{ST}$ & & $F(2.9,55.8)=3.66, p<.02$ & $F(2.2,41)=3.70, p<.04$ & $F(1.6,31.3)=4.24, p<.04$ \\
\hline \multicolumn{5}{|c|}{ Neutral Hit Versus CR } \\
\hline IT & & $F(1,19)=7.42, p<.02$ & $F(1,19)=9.70, p<.006$ & $F(1,19)=30.47, p<.001$ \\
\hline $\mathrm{IT} \times \mathrm{HM}$ & & & & $F(1,19)=8.24, p<.02$ \\
\hline $\mathrm{IT} \times \mathrm{AP}$ & & $F(1.1,21.6)=9.31, p<.005$ & & $F(1.1,21.6)=11.54, p<.003$ \\
\hline $\mathrm{IT} \times \mathrm{ST}$ & & & $F(1.1,21.5)=9.70, p<.004$ & $F(1.2,22.4)=19.75, p<.001$ \\
\hline $\mathrm{IT} \times \mathrm{HM} \times \mathrm{AP}$ & $F(2,37.2)=9.08, p<.001$ & $F(1.8,33.9)=3.57, p<.05$ & $F(1.5,29)=4.37, p<.04$ & $F(1.6,29.9)=3.89, p<.05$ \\
\hline \multicolumn{5}{|c|}{$\mathrm{IT} \times \mathrm{HM} \times \mathrm{AP} \times \mathrm{ST} \quad F(2.5,47.3)=3.69, p<.03$} \\
\hline \multicolumn{5}{|c|}{ Emotional Hit Versus CR } \\
\hline IT & & $F(1,19)=5.96, p<.03$ & $F(1,19)=6.40, p<.03$ & $F(1,19)=30.57, p<.001$ \\
\hline $\mathrm{IT} \times \mathrm{AP}$ & & $F(1.1,21.2)=5.32, p<.03$ & $F(1.3,25)=4.32, p<.04$ & $F(1.5,28.8)=19.41, p<.001$ \\
\hline $\mathrm{IT} \times \mathrm{ST}$ & $F(1.1,20.4)=4.62, p<.05$ & & $F(1.1,20.2)=11.41, p<.003$ & $F(1.1,20.4)=12.44, p<.003$ \\
\hline $\mathrm{IT} \times \mathrm{HM} \times \mathrm{AP}$ & & & $F(1.4,26.7)=4.23, p<.04$ & $F(1.5,29)=4.32, p<.04$ \\
\hline $\mathrm{IT} \times \mathrm{HM} \times \mathrm{ST}$ & & $F(1.4,27.1)=7.39, p<.006$ & $F(1.1,21)=5.86, p<.03$ & $F(1.1,21.1)=5.82, p<.03$ \\
\hline $\mathrm{IT} \times \mathrm{AP} \times \mathrm{ST}$ & & $F(2.7,50.4)=3.23, p<.04$ & & \\
\hline \multicolumn{5}{|c|}{ Emotional Versus Neutral Hits } \\
\hline $\mathrm{IT} \times \mathrm{HM}$ & & & & $F(1,19)=4.87, p<.05$ \\
\hline $\mathrm{IT} \times \mathrm{ST}$ & $F(1.1,21.4)=6.19, p<.02$ & & & \\
\hline $\mathrm{IT} \times \mathrm{HM} \times \mathrm{AP}$ & $F(1.7,32)=3.71, p<.05$ & & & \\
\hline
\end{tabular}

IT = item type (emotional hits, neutral hits, or correct rejections), $\mathrm{HM}=$ hemisphere, $\mathrm{ST}=$ lateral site (inferior, middle, or superior), $\mathrm{AP}=$ chain (frontal, central/temporal, or parietal).

neutral misses over frontal electrodes (see Fig. 4). Subsidiary ANOVAs for the data from frontal, central, and parietal electrodes elucidated this result by revealing that the effect was reliable for the 300- to 500-ms epoch over the frontal electrode chain $[F(1,19)=5.74, p<.02]$.

Emotion effects for hits versus misses

To directly compare the effects of emotion on the ERPs elicited by hits and misses, additional ANOVAs were performed incorporating a factor consisting in the difference between the magnitudes of emotional and neutral hits and of emotional and neutral misses (i.e., emotional hits minus neutral hits and emotional misses minus neutral misses). From here on, we will refer to this factor as EmotionalNeutral Difference.

ANOVAs conducted on the data from the 200- to $300-\mathrm{ms}$, 300 - to $500-\mathrm{ms}, 500-$ to $800-\mathrm{ms}$, and $800-$ to $1,100-\mathrm{ms}$ latencies revealed interactions among the factors Emotional-Neutral Difference and Anterior-Posterior Chain for the 200- to 300-ms and the 300- to 500-ms latency regions $[F(1.2,23.5)=6.99, p<.01$, and $F(1.3,24.5)=$ $8.18, p<.005$, respectively]. Subsidiary ANOVAs conducted separately in the anterior-, central-, and posteriorchain electrodes failed, however, to reveal any effect of emotional-neutral difference on these epochs (i.e., 200300 and 300-500 ms).

For the 800 - to $1,100-\mathrm{ms}$ latency region, ANOVAs revealed a main effect of emotional-neutral difference $[F$ $(1,19)=5.57, p<.029]$ and an interaction between this factor and hemisphere $[F(1,19)=6.23, p<.022]$. These effects reflect the fact that when all electrode locations were taken into account, hits exhibited negative emotion effects (i.e., emotional hits elicited more negative effects than did neutral hits), while misses exhibited positive emotion effects (emotional misses elicited more positive effects than did 
Table 3 Contrast of ERPs between misses and correct rejections (CRs) at different topographic sites and for different latency regions

\begin{tabular}{|c|c|c|c|c|}
\hline \multirow[t]{2}{*}{ Effect } & \multicolumn{4}{|l|}{ Latency Regions (ms) } \\
\hline & $200-300$ & $300-500$ & $500-800$ & $800-1,100$ \\
\hline \multicolumn{5}{|c|}{ All Misses Versus CR } \\
\hline $\mathrm{IT} \times \mathrm{HM}$ & $F(1.8,34.6)=3.63, p<.05$ & & & $F(1.9,35.7)=7.02, p<.004$ \\
\hline $\mathrm{IT} \times \mathrm{AP}$ & $F(2.1,40.6)=6.87, p<.003$ & $F(2.1,40)=4.66, p<.02$ & & $F(1.9,36.5)=3.51, p<.05$ \\
\hline \multicolumn{5}{|c|}{ Neutral Misses Versus CR } \\
\hline $\mathrm{IT} \times \mathrm{HM}$ & & & & $F(1,19)=5.70, p<.03$ \\
\hline \multicolumn{5}{|c|}{ Emotional Misses Versus CR } \\
\hline IT & & $F(1,19)=5.23, p<.04$ & & \\
\hline $\mathrm{IT} \times \mathrm{HM}$ & $F(1,19)=6.51, p<.02$ & $F(1,19)=7.63, p<.02$ & $F(1,19)=8.25, p<.02$ & $F(1,19)=15.36, p<.002$ \\
\hline $\mathrm{IT} \times \mathrm{AP}$ & $F(1.2,23.5)=9.45, p<.003$ & $F(1.3,24.2)=9.81, p<.003$ & $F(1.4,25.7)=4.34, p<.04$ & $F(1.4,27.2)=9.96, p<.003$ \\
\hline \multicolumn{5}{|c|}{ Emotional Versus Neutral Misses } \\
\hline $\mathrm{IT} \times \mathrm{AP}$ & $F(1.2,22.2)=8.43, p<.006$ & $F(1.2,22.6)=6.72, p<.02$ & $F(1.2,22.3)=5.33, p<.03$ & \\
\hline
\end{tabular}

IT = item type (emotional misses, neutral misses, or correct rejections), $\mathrm{HM}=$ hemisphere, $\mathrm{AP}=$ chain (frontal, central/temporal, or parietal)

neutral misses). Subsidiary ANOVAs conducted separately on electrodes from the right and left hemispheres revealed that while no significant effects were found on the right hemisphere $[F(1,19)=2.05, p>.166]$, a main effect of emotional-neutral difference $[F(1,19)=7.94, p<.011]$ was found on the left hemisphere, indicating that the different pattern exhibited by the emotion effects for hits and misses in the 800 - to $1,100-\mathrm{ms}$ epoch was more prominent for the left hemisphere.

\section{Topographic analyses}

The topographies of the emotional and neutral old-new effects for the latency regions in which there were reliable interactions involving item type in the magnitude analyses are illustrated in Fig. 5. All topographic analyses were based on data from the same electrode groupings and site factors that had been used in the initial analyses (see Fig. 2) and were range-normalized in order to eliminate the potentially confounding effects of global amplitude differences (McCarthy \& Wood, 1985). The ANOVA contrasting the latency regions and the scalp topographies of the emotional and neutral old-new effects (i.e., emotional hits minus correct rejections and neutral hits minus correct rejections) - a factor that will be referred to as Old-New Difference from here on-revealed an interaction among latency region, oldnew difference, and site $[F(2.3,44.2)=7.67, p<.001]$, indicating that the topographies of the emotional and neutral old-new effects evolved differentially over time. Subsidiary ANOVAs conducted separately on the data from each latency region showed an interaction among old-new difference and site for the 200 - to 300 -ms epoch only $[F(1.1,21.1)=$ $7.86, p<.009]$. The topographies of the emotional effects (emotional hits - neutral hits) are illustrated in Fig. 5 for the latency regions in which magnitude effects were significant. We found no significant effects involving latency regions and site factors, and thus no evidence that the scalp distributions of the ERP emotion effects elicited by hits varied as a function of time.

The scalp topographies showing the differences between emotional misses versus correct rejections, neutral misses versus correct rejections, and emotional misses versus neutral misses for the latency regions in which significant effects were found in the magnitude analysis are illustrated in Fig. 6. As for the old-new effects described above, an ANOVA was performed to contrast the differences between emotional misses and correct rejections versus neutral misses and correct rejections. A separate ANOVA was also conducted to contrast the differences between emotional misses and neutral misses. We found no significant effects involving the Latency Region and Site factors, and therefore no evidence that the scalp topographies of the ERP emotion effects elicited by misses change as a function of time.

An ANOVA contrasting the scalp topographies of the emotion effects elicited by hits and misses (i.e., the factor termed Emotional-Neutral Difference) for the latency regions in which the magnitude analysis showed emotion effects for either hits or misses revealed an interaction among latency region, emotional-neutral difference, and site $[F(2.9,54.2)=3.18, p<.03]$, indicating that the scalp distributions of the two types of emotion effect differentially varied with time. Subsidiary ANOVAs conducted separately on the data from each latency region revealed an interaction between emotional-neutral difference and anterior-posterior chain for the 200- to 300- and 300- to 500-ms latency regions $[F(1.2,22.9)=5.31, p<.05$, and $F(1.3,24.1)=$ $6.26, p<.025$, respectively], as well as an interaction between emotional-neutral difference and hemisphere for 
Fig. 5 Scalp topographies showing the differences in mean amplitudes for the eventrelated potentials associated with neutral hits versus correct rejections (A), emotional hits versus correct rejections $(\mathbf{B})$, and emotional versus neutral hits $(\mathbf{C})$. Only regions in which ANOVAs showed significant effects on the magnitude data are plotted here. The range in microvolts is indicated under each plot, and the latency regions are at the top. The maximal positivity is indicated by dark red, and the maximal negativity is indicated by dark blue (the nose is at the top of each plot)
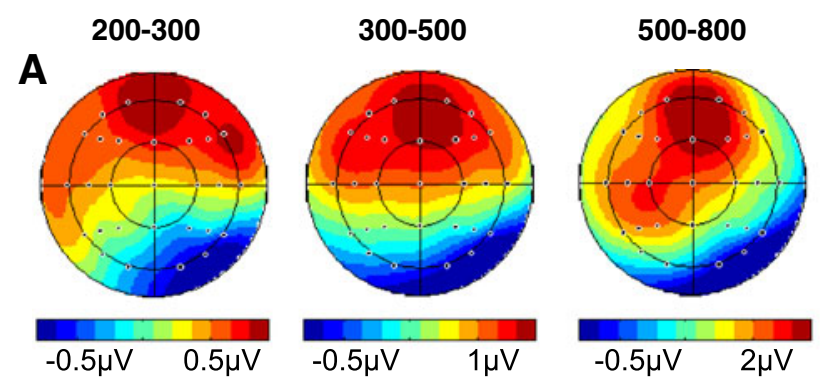

800-1100
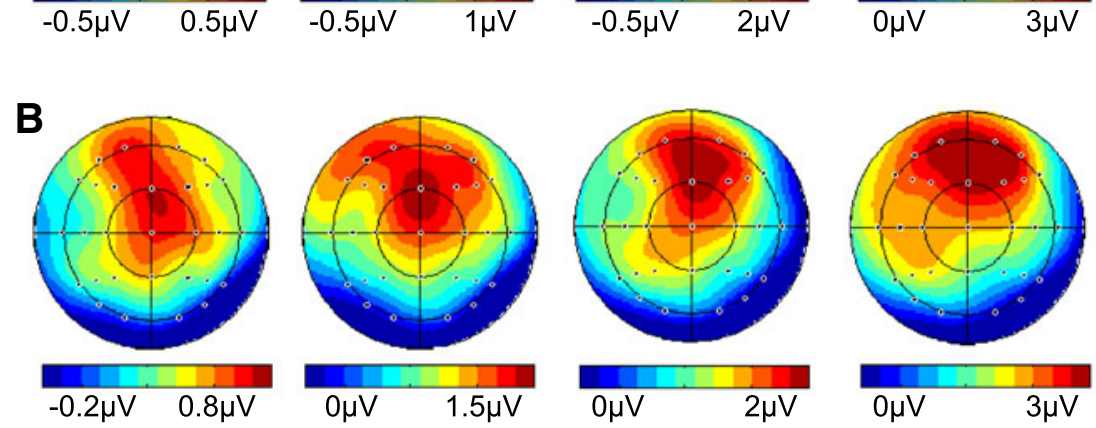

C
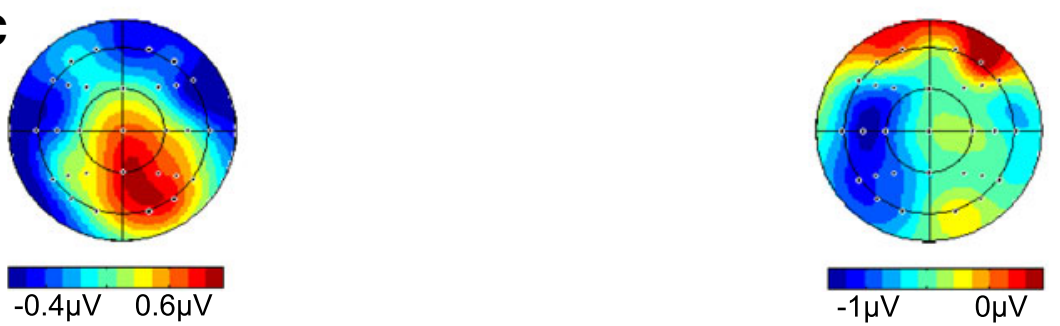

the 800 - to 1,100 -ms latency region $[F(1,19)=6.28, p<.05]$. As can be seen in Figs. 5C and 6C, these interactions reflect the fact that, whereas the emotion effects elicited by misses took the form of an early-onsetting frontal positivity, the emotion effects elicited by hits were largely the result of a later-onsetting, left-lateralized negativity.

\section{Discussion}

\section{Behavioral data}

There were no differences in the accuracy with which test items paired with emotionally negative and emotionally neutral contexts were recognized. This finding may seem surprising, in light of the literature showing memory enhancement for emotionally negative information (Kensinger, 2007), but it is consistent with the results of Smith et al. (2004) and Jaeger et al. (2009), in whose studies null effects for the retrieval of items associated with emotionally negative relative to emotionally neutral contexts were also reported (see also Erk, Kiefer, Grothe, Wunderlich, Spitzer, Walter, 2003; Smith et al., 2005). As was noted by Smith et al. (2004), one explanation for the present findings is that the encoding of emotionally neutral components of an episode (the study items, in the present case) suffers when attention is captured by emotionally arousing components (the scenes on which the items were superimposed; see also Mather, 2007, and Kensinger, Garoff-Eaton \& Schacter, 2007). Clearly, however, this does not mean that at the neural level, the processing of the study items was unaffected by the emotional properties of their contexts (e.g., Jaeger et al., 2009; Smith et al., 2004; see below). In any case, the absence of behavioral differences between the test items associated with the different classes of study contexts simplifies the interpretation of the ERP effects discussed below; that is, these analyses are unlikely to be confounded by such factors as differences in retrieval effort or accuracy.

\section{ERP data}

Consistent with numerous prior reports (for a review, see Rugg \& Curran, 2007), successfully recognized items elicited more positive-going ERPs than did correctly rejected items. These "old-new" effects were evident as early as $200 \mathrm{~ms}$ post-stimulus-onset, and an analysis restricted to the 300 - to $500-\mathrm{ms}$ and 500 - to 800 -ms regions demonstrated that the effects were equally evident for both emotional and neutral hits. We interpret them as examples of the "midfrontal" and "parietal" old-new effects described in numerous prior studies of recognition memory (e.g., Curran, 2000; Rugg et al., 1998; Wilding, 2000; see Rugg \& Curran, 2007, 
Fig. 6 Scalp topographies showing the differences in

A with neutral misses versus correct rejections (A), emotional misses versus correct rejections (B), and emotional versus neutral misses (C). Only regions in which ANOVAs showed significant effects on the magnitude data are plotted here. The range in microvolts is indicated under each plot, and the latency regions are at the top. The maximal positivity is indicated by dark red, and the maximal negativity is indicated by dark blue (the nose is at the top of each plot)

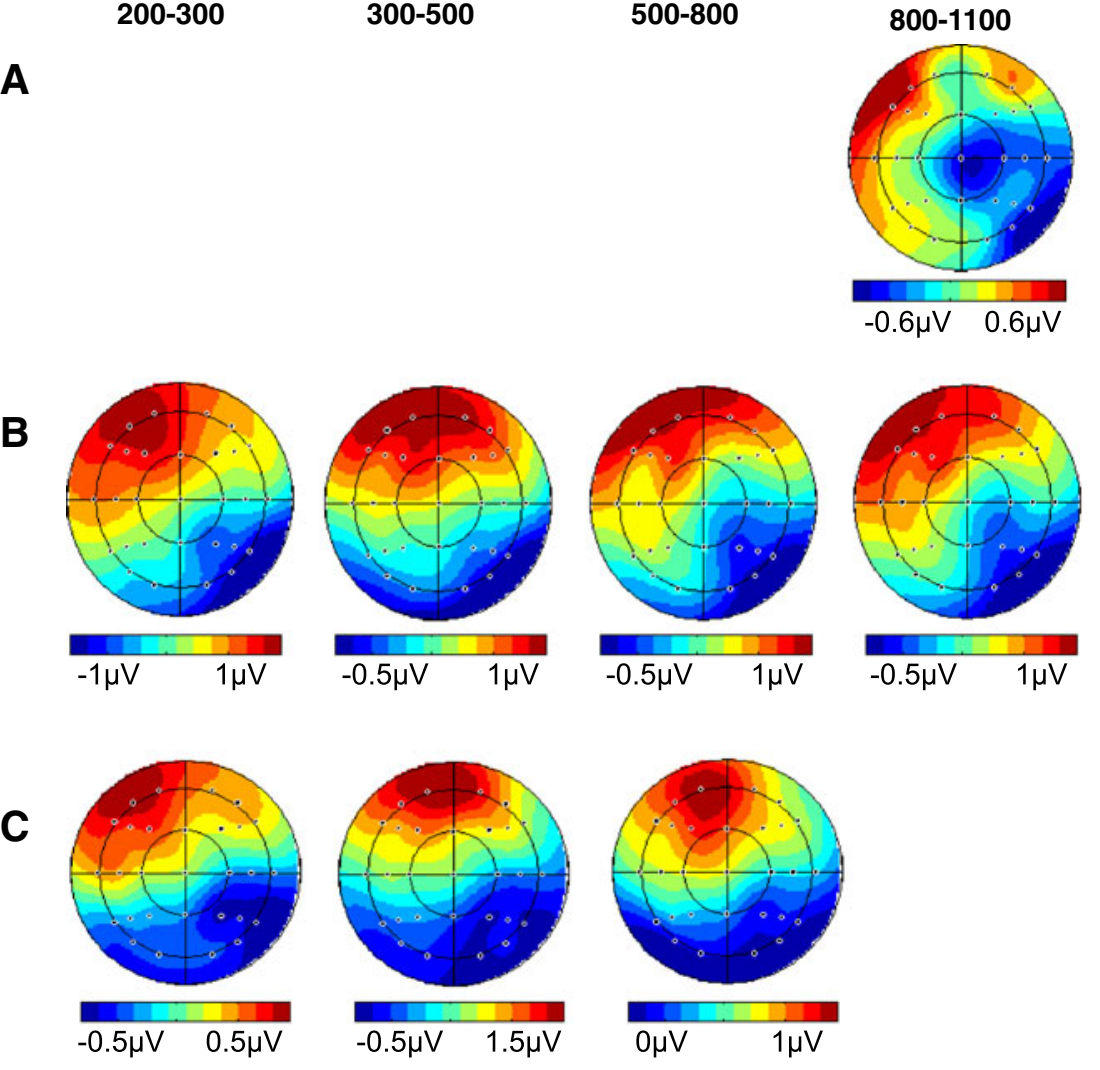

B for a review). Within the framework of dual-process models of recognition memory, the midfrontal old-new effect is held to be a neural correlate of an acontextual sense of familiarity, whereas the parietal effect is a correlate of the successful retrieval of contextual information (recollection; Rugg \& Curran, 2007). From this perspective, given that the two effects did not differ as a function of the emotional properties of the test items' study contexts, neither familiarity nor recollection appears to have been modulated by the study manipulation.

The direct contrast between emotional and neutral hits identified differences in retrieval-related neural activity in the 200- to $300-\mathrm{ms}$ and $800-$ to $1,100-\mathrm{ms}$ latency regions. The earlier effect took the form of increased positivity for emotional relative to neutral hits at parietal electrodes. This effect is strongly reminiscent of the early effects described by Smith et al. (2004) and Jaeger et al. (2009), and is similar in its onset latency to the effects reported when ERPs were elicited directly by emotionally arousing versus unarousing stimuli (Cuthbert et al., 2000; Keil et al., 2002; Schupp et al., 2000). The effect in the 800 - to 1,100-ms latency region was reversed in polarity relative to that in the earlier latency region and was maximal over the left hemisphere (see Figs. 3 and 5). This effect bears some similarity to the effect reported in our prior study (Jaeger et al., 2009), although that effect had an earlier onset and was more sustained over the parietal scalp. One account of the difference between the present effect and the one reported by Jaeger et al. is that in our prior study an additional test phase was conducted shortly after the study phase. That is, while in the present study all of the test items were presented $24 \mathrm{~h}$ after the study phase, in Jaeger et al.'s study half of the test items were presented $10 \mathrm{~min}$ after they had been studied, and the other half $24 \mathrm{~h}$ later. The intermixing in our previous study of items with very disparate memory strengths may have led the items tested after $24 \mathrm{~h}$ to be processed somewhat differently than those in the present study.

The outcome of the contrast between misses and correct rejections differed according to the study context of the test item. In the 800- to 1,100-ms latency region, missed items from neutral contexts elicited a more positive waveform over the left hemisphere than did correct rejections, while demonstrating the opposite pattern over the right hemisphere (Fig. 4). Misses from emotional contexts, by contrast, elicited more positive waveforms relative to correct rejections at frontal sites in all latency regions. The earliest effects of study context on the ERPs elicited by misses differed from the effects elicited by recognized test items in terms of both their magnitudes and, more importantly, their topographies. For the reasons noted in the introduction, this topographical dissociation is difficult to reconcile with the proposal that the effects reflect emotionally arousing properties of the test items acquired through association with their study contexts. Rather, the findings point to an electrophysiological dissociation between the neural correlates 
of explicit and implicit retrieval as a function of the emotional properties of the contexts in which the items were studied.

The contrast between emotional misses and correct rejections revealed effects with the same polarity and within a similar time frame to those found in a prior study, in which implicit memory was operationalized by studied items misjudged as new (Rugg et al., 1998, Exp. 1). Those authors reported a positive effect during the 300- to 500-ms latency region for unrecognized old items contrasted with new items. However, while those prior effects were largest over parietal electrode sites, the present effects were maximal at frontal sites. One possible account of these topographical differences is that the posterior effects reported by Rugg et al. reflected neural activity engaged during the implicit retrieval of words (see also Friedman, 2004; Paller \& Gross, 1998; Paller, Kutas, \& McIsaac, 1998; Woollams, Taylor, Karayanidis, \& Henson, 2008), whereas the present effects elicited by emotional misses reflected neural activity associated with the implicit retrieval of pictorial information (Voss \& Paller, 2007, 2009; Voss et al., 2010; but see Li et al., 2008).

While the present implicit emotional effects partially resemble those described in prior reports of implicit effects for pictorial materials (in that they were frontally distributed and had an onset around $200 \mathrm{~ms}$ poststimulus), the previously reported effects had been negative-going rather than positivegoing (Voss \& Paller, 2007, 2009; Voss et al., 2010). The reason for this discrepancy between those previous studies and the present one is unclear. A possible account might be related to the relatively long study-test delay employed here. In contrast to the short study-test delays employed in prior ERP studies of implicit retrieval, the present study-test delay meant that short-term (synaptic) consolidation would have been complete (McGaugh, 2000), which in turn might have modulated the mechanisms of implicit retrieval (see also Jaeger et al., 2009).

An alternative account of the present ERP effects elicited by unrecognized items is that, rather than reflecting implicit memory, the effects represent neural activity associated with below-criterion explicit retrieval (cf. Bridson, Fraser, Herron, \& Wilding, 2006). According to this argument, the memory signal elicited by missed items was merely too weak to exceed the criterion for an "old" response. According to the response bias index $\mathrm{Br}$ (for which values lower than 0.5 indicate a conservative response bias; Snodgrass \& Corwin, 1988), the response criteria adopted by the present participants were conservative $(\mathrm{Br}=0.15$ for both emotional and neutral items, as opposed to a value of 0.5 for a neutral bias), giving credence to this proposal. One aspect of the present findings that is hard to reconcile with this account, however, is that if the differential emotion ERP effects for hits and misses were driven merely by differences in memory strength, one would expect only quantitative differences between the effects (i.e., differences in their magnitudes, but not in their scalp topographies).
The present data demonstrate instead that the effects of study context for hits and misses differed qualitatively (i.e., in their scalp topographies), making it likely that at least partially distinct neural populations, and hence different cognitive operations, were responsible for the context effects associated with each response category.

In summary, in the present study we investigated ERP effects elicited by recognized and unrecognized studied items encoded in either emotionally negative or emotionally neutral contexts. Crucially, context effects differed qualitatively according to whether the items were or were not correctly judged as old. We interpret these ERP findings as evidence that the manipulation of study context modulated the neural correlates of both explicit and implicit memory.

Author Note A.J. is now at the Department of Psychology, Pontifícia Universidade Católica do Rio Grande do Sul, Porto Alegre, Brazil. He was supported by CAPES/PDEE. This work was supported by the National Institute of Mental Health, Grant No. R01-MH072966.

Correspondence concerning this article should be addressed to Antonio Jaeger, Pontifícia Universidade Católica do Rio Grande do Sul, Pós-Graduação em Psicologia, Av. Ipiranga, n 6681 , prédio 11, sala 940, CEP 90619-900, Porto Alegre/Rio Grande do Sul. E-mail: antonio.jaeger@gmail.com

\section{References}

American Electroencephalographic Society. (1994). Guidelines for standard electrode position nomenclature. Journal of Clinical Neurophysiology, 11, 111-113.

Bridson, N. C., Fraser, C. S., Herron, J. E., \& Wilding, E. L. (2006). Electrophysiological correlates of familiarity in recognition memory and exclusion tasks. Brain Research, 1114, 149-160.

Buchanan, T. W. (2007). Retrieval of emotional memories. Psychological Bulletin, 133, 761-779.

Christianson, S. A. (1992). The handbook of emotion and memory (pp. 3-108). Hillsdale: Erlbaum.

Curran, T. (2000). Brain potentials of recollection and familiarity. Memory \& Cognition, 28, 923-938.

Cuthbert, B. N., Schupp, H. T., Bradley, M. M., Birbaumer, N., \& Lang, P. J. (2000). Brain potentials in affective picture processing: Covariation with autonomic arousal and affective report. Biological Psychology, 52, 95-111. doi:10.1016/S0301-0511(99)00044-7

Eder, A. B., Leuthold, H., Rothermund, K., \& Schweinberger, S. R. (2012). Automatic response activation in sequential affective priming: An ERP study. Social Cognitive and Affective Neuroscience, 7, 436-445.

Erk, S., Kiefer, M., Grothe, J., Wunderlich, A. P., Spitzer, M., \& Walter, H. (2003). Emotional context modulates subsequent memory effect. NeuroImage, 18, 439-447.

Friedman, D. (2004). ERP studies of recognition memory: Differential effects of familiarity, recollection, and episodic priming. Cognitive Sciences, 1, 81-121.

Hamann, S. B. (2001). Cognitive and neural mechanisms of emotional memory. Trends in Cognitive Sciences, 5, 394-400.

Henson, R. N. A., Rylands, A., Ross, E., Vuilleumier, P., \& Rugg, M. D. (2004). The effect of repetition lag on electrophysiological and haemodynamic correlates of visual object priming. NeuroImage, 21, 1674-1689. doi:10.1016/j.neuroimage.2003.12.020 
Hinojosa, J. A., Carretié, L., Méndez-Bértolo, C., Míguez, A., \& Pozo, M. A. (2009). Arousal contributions to affective priming: Electrophysiological correlates. Emotion, 9, 164-171. doi:10.1037/ a 0014680

Jaeger, A., Johnson, J. D., Corona, M., \& Rugg, M. D. (2009). ERP correlates of incidental retrieval of emotional information: Effects of study-test delay. Brain Research, 1269, 105-113.

Keil, A., Bradley, M. M., Hauk, O., Rockstroh, B., Elbert, T., \& Lang, P. J. (2002). Large-scale neural correlates of affective picture processing. Psychophysiology, 39, 641-649. doi:10.1017/ S0048577202394162

Kensinger, E. A. (2007). Negative emotion enhances memory accuracy. Current Directions in Psychological Science, 16, 213-218.

Kensinger, E. A., Garoff-Eaton, R. J., \& Schacter, D. L. (2007). Effects of emotion on memory specificity: Memory trade-offs elicited by negative visually arousing stimuli. Journal of Memory and Language, 56, 575-591. doi:10.1016/j.jml.2006.05.004

Lang, P. J., Bradley, M. M., \& Cuthbert, B. N. (2008). International affective picture system (IAPS): Affective ratings of pictures and instruction manual. Technical Report A-8. University of Florida, Gainesville, FL.

Li, W., Zinbarg, R. E., Boehm, S. G., \& Paller, K. A. (2008). Neural and behavioral evidence for affective priming from unconsciously perceived emotional facial expressions and the influence of trait anxiety. Journal of Cognitive Neuroscience, 20, 95-107.

Maratos, E. J., Dolan, R. J., Morris, J. S., Henson, R. N. A., \& Rugg, M. D. (2001). Neural activity associated with episodic memory for emotional context. Neuropsychologia, 39, 910-920.

Maratos, E. J., \& Rugg, M. D. (2001). Electrophysiological correlates of the retrieval of emotional and non-emotional context. Journal of Cognitive Neuroscience, 13, 877-891.

Mather, M. (2007). Emotional arousing and memory binding: An object-based framework. Perspectives on Psychological Science, 2, 33-52. doi:10.1111/j.1745-6916.2007.00028.x

McCarthy, G., \& Wood, C. C. (1985). Scalp distribution of eventrelated potentials: An ambiguity associated with analysis of variance models. Electroencephalography and Clinical Neurophysiology, 62, 203-208.

McGaugh, J. L. (2000). Memory-A century of consolidation. Science, 287, 248-251. doi:10.1126/science.287.5451.248

Paller, K. A., \& Gross, M. (1998). Brain potentials associated with perceptual priming vs. explicit remembering during the repetition of visual word-form. Neuropsychologia, 36, 559-571.

Paller, K. A., Kutas, M., \& McIsaac, H. K. (1998). An electrophysiological measure of priming of visual word form. Consciousness and Cognition, 7, 54-66.
Rugg, M. D., \& Curran, T. (2007). Event-related potentials and recognition memory. Trends in Cognitive Sciences, 11, 251257.

Rugg, M. D., Mark, R. E., Walla, P., Schloerscheidt, A. M., Birch, C. S., \& Allan, K. (1998). Dissociation of the neural correlates of implicit and explicit memory. Nature, 392, 595-598.

Schupp, H. T., Cuthbert, B. N., Bradley, M. M., Cacioppo, J. T., Ito, T., \& Lang, P. J. (2000). Affective picture processing: The late positive potential is modulated by motivational relevance. Psychophysiology, 37, 257-261. doi:10.1111/1469-8986.3720257

Smith, A. P. R., Dolan, R. J., \& Rugg, M. D. (2004). Eventrelated potentials correlates of the retrieval of emotional and nonemotional context. Journal of Cognitive Neuroscience, 16 , $760-775$.

Smith, A. P. R., Henson, R. N., Rugg, M. D., \& Dolan, R. J. (2005). Modulation of retrieval processing reflects accuracy of emotional source memory. Learning and Memory, 12, 472-479.

Smith, A. P. R., Stephan, K. E., Rugg, M. D., \& Dolan, R. J. (2006). Task and content modulate amygdala-hippocampal connectivity in emotional retrieval. Neuron, 49, 631-638.

Snodgrass, J. G., \& Corwin, J. (1988). Pragmatics of measuring recognition memory: Applications to dementia and amnesia. Journal of Experimental Psychology. General, 117, 34-50. doi:10.1037/ 0096-3445.117.1.34

Tapia, M., Carretié, L., Sierra, B., \& Mercado, F. (2008). Incidental encoding of emotional pictures: Affective bias studied through event related brain potentials. International Journal of Psychophysiology, 68, 193-200.

Voss, J. L., \& Paller, K. A. (2007). Neural correlates of conceptual implicit memory and their contamination of putative neural correlates of explicit memory. Learning \& Memory, 14, 259-267.

Voss, J. L., \& Paller, K. A. (2009). An electrophysiological signature of unconscious recognition memory. Nature Neuroscience, 12, 349355.

Voss, J. L., Schendan, H. E., \& Paller, K. A. (2010). Finding meaning in novel geometric shapes influences electrophysiological correlates of repetition and dissociates perceptual and conceptual priming. Neurolmage, 49, 2879-2889.

Wilding, E. L. (2000). In what way does the parietal ERP old/new effect index recollection? International Journal of Psychophysiology, 35, 81-87.

Woollams, A. M., Taylor, J. R., Karayanidis, F., \& Henson, R. (2008). Event-related potentials associated with masked priming of test cues reveal multiple potential contributions to recognition memory. Journal of Cognitive Neuroscience, 20, 1114-1129. 